\title{
Higgs Resonance Studies At The First Muon Collider
}

\author{
Basim Kamal, William J. Marciano and Zohreh Parsa
}

Brookhaven National Laboratory, Upton, New York 11973

\begin{abstract}
Higgs resonance signals and backgrounds at the First Muon Collider are discussed. Effects due to beam polarization and background angular distributions (forward-backward charge asymmetries) are examined. The utility of those features for improving precision measurements and narrow resonance "discovery" scans is described.
\end{abstract}

If the standard model Higgs boson has a mass $\lesssim 160 \mathrm{GeV}$ (i.e. below the $W^{+} W^{-}$ decay threshold), it will have a very narrow width and can be resonantly studied in the $s$-channel via $\mu^{-} \mu^{+} \rightarrow H$ production at the First Muon Collider (FMC). Within the framework of supersymmetry or more general two Higgs doublet scenarios, there can be several neutral spin zero bosons; $h, H$, and $A$, all of which might be resonantly produced. The lightest scalar, $h$, of supersymmetry is expected to be $\lesssim 150$ $\mathrm{GeV}$ (and narrow), with the range 80-130 GeV favored. Precision electroweak measurements also tend to suggest, via quantum loop sensitivity, a relatively light Higgs. Hence, there are strong motivations to examine the capabilities of the FMC for producing and studying relatively light scalar resonances $[1,2]$.

A strategy for "light" Higgs physics studies would be to first discover the Higgs particle at LEPII, the Tevatron, or the LHC and then thoroughly scrutinize its properties on resonance at the FMC. There, one would hope to precisely determine the Higgs mass, width, and primary decay rates [3]. Besides those interesting physics studies, such an initiative would provide a nice testing ground for muon collider technology and lay the foundation for future much higher energy facilities.

The FMC Higgs resonance program would entail two stages: 1) "Discovery" via an energy scan which pinpoints the precise resonance position and (perhaps) determines its width. Since pre-FMC efforts may only determine the Higgs mass to $\mathcal{O}(200 \mathrm{MeV})$ or worse and its width is expected to be narrow $\mathcal{O}(1 \sim 30 \mathrm{MeV})$ for $m_{H} \lesssim 160 \mathrm{GeV}$, the resonance scan may be very time consuming [3]. 2) Precision measurements of the primary Higgs decay modes. Deviations from standard model expectations could point to additional Higgs structure or elucidate the framework 
TABLE 1. Expected signals and backgrounds (fully integrated) for a standard model Higgs with $m_{H}=110 \mathrm{GeV}, \Gamma_{H} \simeq 3 \mathrm{MeV}$. Muon collider resonance conditions with no polarization, $\Delta E / E \simeq 3 \times 10^{-5}$, and $L=0.05$ $\mathrm{fb}^{-1}$ are assumed. The total number of Higgs scalars produced is $\sim 3000$. Realistic efficiency and acceptance cuts are likely to dilute signal and backgrounds for $b \bar{b}$ and $c \bar{c}$ by a 0.5 factor.

\begin{tabular}{lccc}
\hline$H \rightarrow$ & $b \bar{b}$ & $c \bar{c}$ & $\tau \bar{\tau}$ \\
\hline$N_{S}$ (events) & 2400 & 210 & 270 \\
$N_{B}$ (events) & 2520 & 2416 & 945 \\
$\pm \sqrt{N_{S}+N_{B}} / N_{S}$ & \pm 0.03 & \pm 0.24 & \pm 0.13 \\
\hline
\end{tabular}

of supersymmetry [3]. (Expectations for $m_{H}=110 \mathrm{GeV}$ are illustrated in Table 1.)

The Higgs resonance "discovery" capability and scan time will depend on $N_{S} / \sqrt{N_{B}}$, where $N_{S}$ is the Higgs signal and $N_{B}$ is the expected background. The precision measurement sensitivity will be determined by $N_{S} / \sqrt{N_{B}+N_{S}}$. For both, it will be extremely important to enhance the signal and suppress backgrounds as much as possible. To that end, one should employ highly resolved $\mu^{+} \mu^{-}$beams with a very small energy spread. The proposed $\Delta E / E \simeq 3 \times 10^{-5}$ is well matched to the narrow Higgs width. It allows $N_{S} / N_{B} \sim \mathcal{O}(1)$ for the primary $H \rightarrow b \bar{b}$ mode (see Table 1). Unfortunately, high resolution is accompanied by luminosity loss. The current goal of $\mathcal{L}_{\text {ave }} \simeq 5 \times 10^{30} \mathrm{~cm}^{-2} \mathrm{~s}^{-1}$ on resonance is probably not ambitious enough. One should strive for another order of magnitude in luminosity while maintaining the outstanding beam energy resolution.

In this paper, we examine two additional ways of enhancing the Higgs signal to background ratio: beam polarization and final state angular distributions. The Higgs signal $\mu^{-} \mu^{+} \rightarrow H \rightarrow f \bar{f}$ results from left-left (LL) or right-right (RR) beam polarizations and leads to an isotropic (i.e. constant) $f \bar{f}$ signal in $\cos \theta$ (the angle between the $\mu^{-}$and $f$ ). Standard model backgrounds $\mu^{-} \mu^{+} \rightarrow \gamma^{*}$ or $Z^{*} \rightarrow f \bar{f}$ result from LR or RL initial state polarizations and give rise to $\left(1+\cos ^{2} \theta+\right.$ $\left.\frac{8}{3} A_{F B} \cos \theta\right)$ angular distributions. Similar statements apply to $W W^{*}$ and $Z Z^{*}$ final states, but those modes will not be discussed here.

To illustrate the difference between signal, $\mu^{-} \mu^{+} \rightarrow H \rightarrow f \bar{f}$, and background, $\mu^{-} \mu^{+} \rightarrow \gamma^{*}$ or $Z^{*} \rightarrow f \bar{f}$, we give the combined differential production rate with respect to $x \equiv \cos \theta=4 \mathbf{p}_{\mu^{-}} \cdot \mathbf{p}_{\mathbf{f}} / s$ for polarized muon beams and fixed luminosity

$$
\begin{aligned}
\frac{d N\left(\mu^{-} \mu^{+} \rightarrow f \bar{f}\right)}{d x} & =\frac{1}{2} N_{S}\left(1+P_{+} P_{-}\right) \\
& +\frac{3}{8} N_{B}\left[1-P_{+} P_{-}+\left(P_{+}-P_{-}\right) A_{L R}\right]\left(1+x^{2}+\frac{8}{3} x A_{e f f}\right) .
\end{aligned}
$$

$P_{+}\left(P_{-}\right)$is the $\mu^{+}\left(\mu^{-}\right)$polarization with $P=-1$ pure left-handed, $P=+1$ pure right handed, and $P=0$ unpolarized. $N_{S}$ is the fully integrated $(-1<x \leq 1)$ 


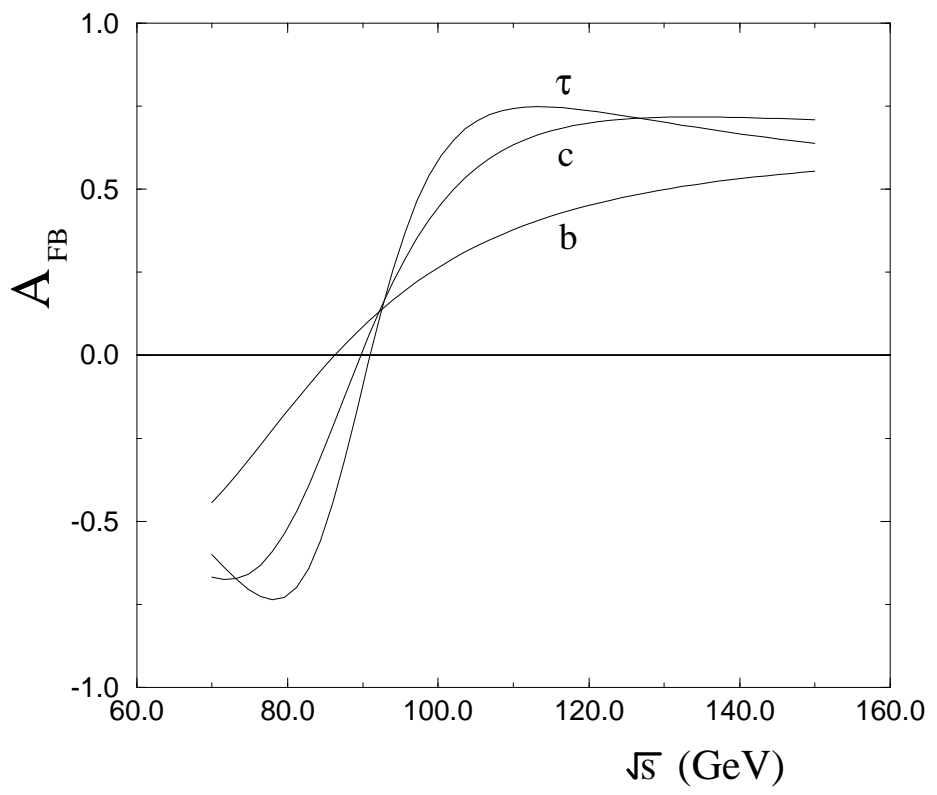

FIGURE 1. Forward-backward asymmetry for $\mu^{-} \mu^{+} \rightarrow f \bar{f}$.

Higgs signal and $N_{B}$ the integrated background for the case of unpolarized beams, $P_{+}=P_{-}=0$. In that general expression,

$$
A_{L R} \equiv \frac{\sigma_{L R \rightarrow L R}+\sigma_{L R \rightarrow R L}-\sigma_{R L \rightarrow R L}-\sigma_{R L \rightarrow L R}}{\sigma_{L R \rightarrow L R}+\sigma_{L R \rightarrow R L}+\sigma_{R L \rightarrow R L}+\sigma_{R L \rightarrow L R}},
$$

where, for example, $L R \rightarrow L R$ stands for $\mu_{L}^{-} \mu_{R}^{+} \rightarrow f_{L} \bar{f}_{R}$. The effective forwardbackward asymmetry is given by

$$
A_{e f f}=\frac{A_{F B}+P_{e f f} A_{L R}^{F B}}{1+P_{e f f} A_{L R}}
$$

with

$$
\begin{aligned}
P_{\text {eff }} & =\frac{P_{+}-P_{-}}{1-P_{+} P_{-}}, \\
A_{F B} & =\frac{3}{4} \frac{\sigma_{L R \rightarrow L R}+\sigma_{R L \rightarrow R L}-\sigma_{L R \rightarrow R L}-\sigma_{R L \rightarrow L R}}{\sigma_{L R \rightarrow L R}+\sigma_{R L \rightarrow R L}+\sigma_{L R \rightarrow R L}+\sigma_{R L \rightarrow L R}}, \\
A_{L R}^{F B} & =\frac{3}{4} \frac{\sigma_{L R \rightarrow L R}+\sigma_{R L \rightarrow L R}-\sigma_{L R \rightarrow R L}-\sigma_{R L \rightarrow R L}}{\sigma_{L R \rightarrow L R}+\sigma_{R L \rightarrow L R}+\sigma_{L R \rightarrow R L}+\sigma_{R L \rightarrow R L}} .
\end{aligned}
$$

and the $\mu_{i}^{-} \mu_{j}^{+} \rightarrow f_{i^{\prime}} \bar{f}_{j^{\prime}}$, cross sections $(i \neq j)$ are to lowest order

$$
\sigma_{i j \rightarrow i^{\prime} j^{\prime}}=\left(N_{C}\right) \sigma_{0}\left[1-\frac{s}{m_{W}^{2}}\left(1+T_{3 \mu_{i}}-\frac{T_{3 f_{i^{\prime}}}}{Q_{f}}\left(1+\frac{T_{3 \mu_{i}}}{\sin ^{2} \theta_{W}}\right)\right)\right]^{2},
$$




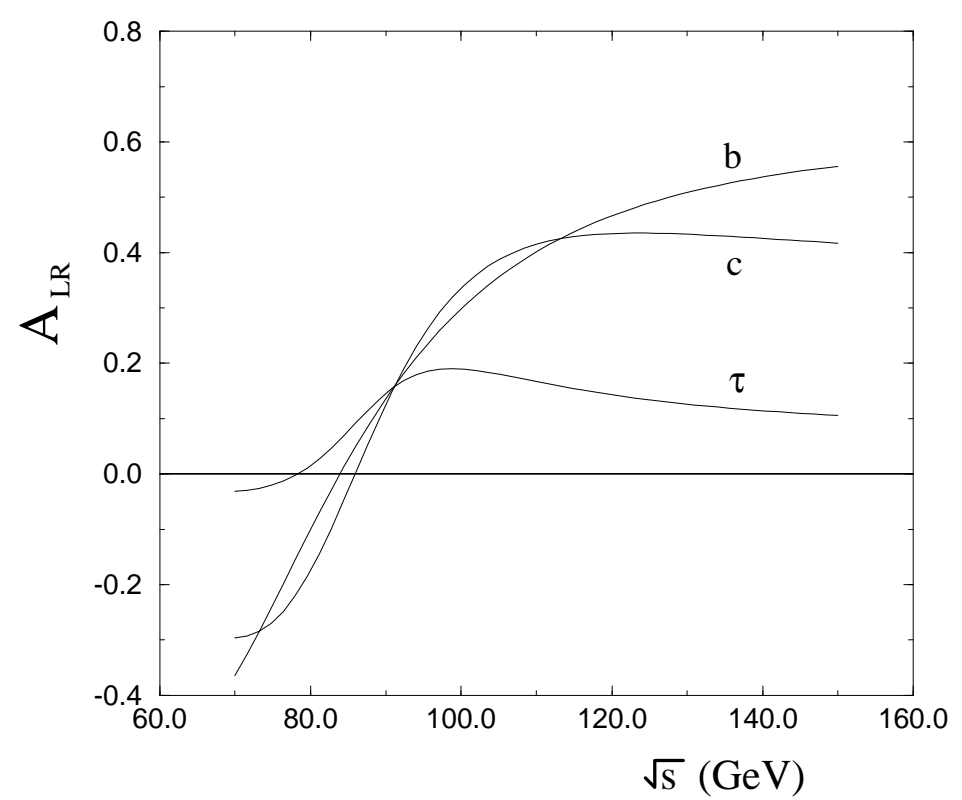

FIGURE 2. Left-right asymmetry for $\mu^{-} \mu^{+} \rightarrow f \bar{f}$.

$$
\begin{aligned}
& T_{3 \mu_{L}}=T_{3 \tau_{L}}=T_{3 b_{L}}=-T_{3 c_{L}}=-1 / 2, \\
& T_{3 f_{R}}=0, \quad Q_{\tau}=3 Q_{b}=-\frac{3}{2} Q_{c}=-1 \quad\left(N_{C}=3 \text { for } f=b, c\right) .
\end{aligned}
$$

Realistic cuts, efficiencies, systematic errors etc, will not be considered. They are likely to dilute the $b \bar{b}$ and $c \bar{c}$ event rates by a factor of 0.5 . In addition, we ignore the radiative $Z$ production tail under the assumption such events are vetoed.

The (unpolarized) forward-backward asymmetries are illustrated in Fig. 1. Note that $A_{F B}$ is large (near maximal) for $\tau \bar{\tau}$ and $c \bar{c}$ in the region of interest. As we shall see, that feature can help in discriminating signal from background.

In principle, large polarization can be important for enhancing "discovery" and precision measurement sensitivity for the Higgs. From Eq. (1), we find that $N_{S} / \sqrt{N_{B}}$ is enhanced ( for integrated signal and background) by the factor

$$
\kappa_{\mathrm{pol}}=\frac{1+P_{+} P_{-}}{\sqrt{1-P_{+} P_{-}+\left(P_{+}-P_{-}\right) A_{L R}}},
$$

where the $A_{L R}$ are shown in Fig. 2. That result generalizes the $P_{+}=P_{-}$case [4]. For natural beam polarization [1], $P_{+}=P_{-}=0.2$ (assuming spin rotation of one beam), the enhancement factor is only 1.06. For larger polarization, $P_{+}=$ $P_{-}=0.5$, one obtains a 1.44 enhancement factor (statistically equivalent to about a factor of 2 luminosity increase). Unfortunately, obtaining 0.5 polarization simply by muon energy cuts reduces each beam intensity [1] by a factor of $1 / 4$, resulting in a 


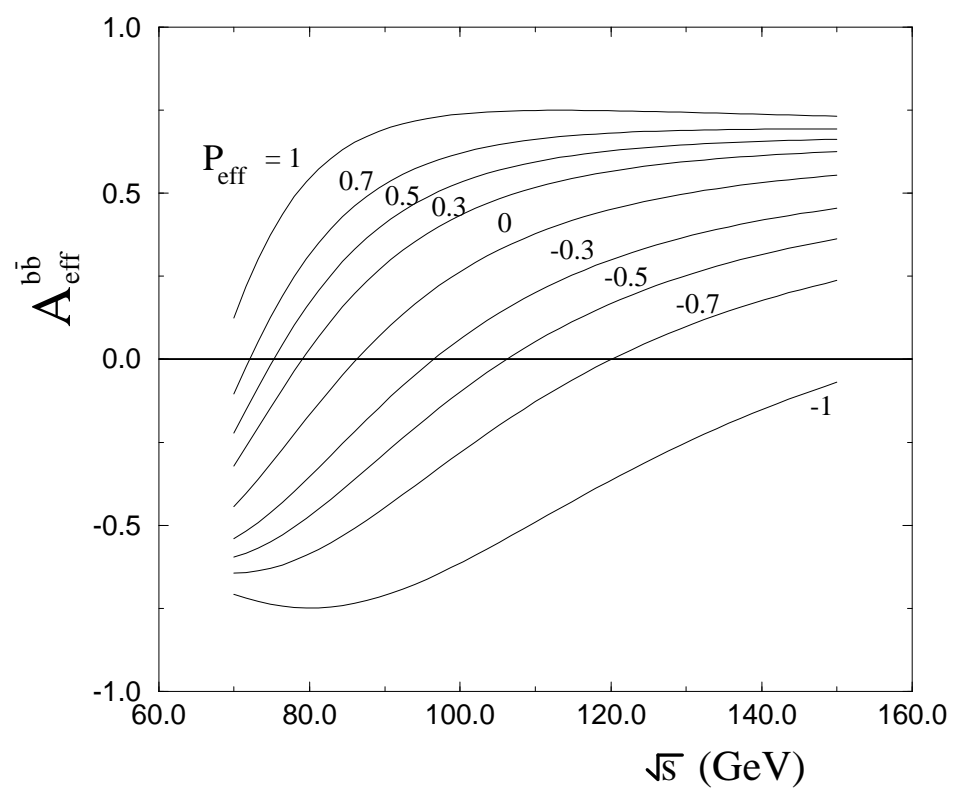

FIGURE 3. Effective forward-backward asymmetry for $\mu^{-} \mu^{+} \rightarrow b \bar{b}$.

luminosity reduction by $1 / 16$. Such a tradeoff is clearly unacceptable. Polarization will be a useful tool in Higgs resonance studies only if high polarization is achievable with little luminosity loss. Tau final state polarizations can also be used to help improve the $H \rightarrow \tau \bar{\tau}$ measurement, but will not be discussed here.

Some "discovery" or sensitivity enhancement can also be obtained from angular discrimination. A proper study would include detector acceptance cuts and maximum likelihood fits. Here, we wish to only crudely approximate the gain. For that purpose, we assume perfect (infinitesimal) binning and obtain the measurement sensitivity enhancement factor

$$
\frac{1}{2}\left(1+P_{+} P_{-}\right) \sqrt{N_{S}+N_{B}}\left[\int \frac{d x}{d N / d x}\right]^{1 / 2}
$$

which becomes, from Eq. (1),

$$
\kappa_{\mathrm{pol}} \sqrt{\frac{2}{3}} \sqrt{\frac{N_{S}+N_{B}}{N_{B}}}\left(\frac{\tan ^{-1}\left(\frac{2}{\zeta} \sqrt{1-\frac{16}{9} A_{e f f}^{2}+\zeta}\right)}{\sqrt{1-\frac{16}{9} A_{e f f}^{2}+\zeta}}\right)^{1 / 2}, \quad \zeta \equiv \frac{4}{3} \frac{N_{S}}{N_{B}} \frac{\kappa_{\mathrm{pol}}^{2}}{1+P_{+} P_{-}} .
$$

For $A_{\text {eff }} \simeq 3 / 4, \zeta \simeq 0.38$ (which roughly applies to $\tau \bar{\tau}$ ) and $P_{+}=P_{-}=0$, one finds a sensitivity enhancement of 1.33 . That means the $\pm 13 \%$ statistical error in Table 1 would be reduced to $\pm 10 \%$. Similar sensitivity enhancements apply to $c \bar{c}$. In the case of $H \rightarrow b \bar{b}$, the primary discovery mode, $A_{\text {eff }} \simeq 0.4$ and one finds only a $3 \%$ 
enhancement. One can increase the effective $b \bar{b}$ forward-backward asymmetry via polarization (see Fig. 3). However, one must again confront the issue of luminosity loss.

In the case of "discovery", a very large forward-backward asymmetry (near maximal) can, in principle, significantly reduce the scan time. For the highly idealized coverage and binning assumed above, the time is reduced by the factor

$$
\frac{1}{\kappa_{\mathrm{pol}}^{2}} \frac{3}{\pi} \sqrt{1-\frac{16}{9} A_{\text {eff }}^{2}} .
$$

Of course, that naive formula must be corrected for realistic acceptances, efficiencies, etc.; so, it should not be taken too literally (particularly for $A_{\text {eff }} \simeq 3 / 4$ ). Nevertheless, applying it to the $b \bar{b}$ discovery mode with "natural" $P_{+}=P_{-}=0.2$ and $A_{e f f} \simeq 0.37$ gives a scan reduction time factor of 0.74 .

The $H \rightarrow \tau \bar{\tau}$ "discovery" time is about 15 times longer than that of the $b \bar{b}$ (with efficiencies) for fully integrated signals. Employing $A_{F B} \simeq 0.743$ and assuming tau detection down to about $15^{\circ}$ from the beams, reduces that time by about a factor of $6 \sim 7$, making it somewhat less than $1 / 2$ as effective as $b \bar{b}$. Using both together along with all background angular information should, therefore, reduce the scan time by almost a factor of 2 compared to using the integrated $b \bar{b}$ signal alone. Such a reduction would be extremely welcome, particularly if the luminosity is less than expected.

In conclusion, we have shown that polarization is potentially useful for Higgs resonance studies, but only if the accompanying luminosity reduction is not significant. Large forward-backward asymmetries can also be used to enhance the Higgs "discovery" signal or improve precision measurements, particularly for $\tau \bar{\tau}$. However, to make the s-channel Higgs "factory" a compelling facility, one must focus on attaining the outstanding beam resolution assumed here and maintaining the highest luminosity possible.

\section{REFERENCES}

1. Muon Collider Feasibility Study, BNL Report 52503 (1996).

2. Cline, D., "The Problems and Physics Prospects for a $\mu^{+} \mu^{-}$Collider", in Future High Energy Colliders, edited by Z. Parsa, AIP Conference Proceedings 397, 1997, pp. 203-218.

3. Barger, V., Berger, M.S., Gunion, J.F., and Han, T., "The Physics Capabilities of $\mu^{+} \mu^{-}$Colliders", in Future High Energy Colliders, edited by Z. Parsa, AIP Conference Proceedings 397, 1997, pp. 219-233; Phys. Rep. 286, 1-51 (1997); Phys. Rev. Lett. 75, 1462-1465 (1995).

4. Parsa, Z., (unpublished). 